\title{
CLASSIFICATION OF OPERATORS BY MEANS OF THE OPERATIONAL CALCULUS ${ }^{1}$
}

\author{
BY SHMUEL KANTOROVITZ
}

Communicated by Felix Browder, November 27, 1963

1. Introduction. Let $\boldsymbol{A}=\boldsymbol{A}(\Delta)$ be a topological algebra of complex valued functions defined on a subset $\Delta$ of the complex plane, with the usual operations. Suppose that $A$ contains the restrictions to $\Delta$ of polynomials. Let $B(X)$ be the Banach algebra of all bounded linear operators on the Banach space $X$ into itself. We say that an operator $T$ is of class $\boldsymbol{A}$ (notation: $T \in(\boldsymbol{A})$ ) if there exists a continuous representation $f \rightarrow T(f)$ of $A$ into $B(X)$ such that $T(1)=I$ and $T(z)=T$. Such a representation is called an A-operational calculus for $T$. A class $(\boldsymbol{A})$ may be as wide as $B(X)$ (if $\boldsymbol{A}$ consists of all entire functions with the topology of uniform convergence on every compact), or as narrow as the class of hermitian operators with spectrum in a given compact $\Delta$ (if $\boldsymbol{A}=C(\Delta), T(\cdot)$ is norm decreasing, and $X$ is a Hilbert space). Related approaches are found in $[3 ; 5]$.

2. Restrictions on $\boldsymbol{A}$. Let $H(\Delta)$ denote the algebra of all complex valued functions which are locally holomorphic in a neighborhood of $\Delta$, with the usual topology.

Condition 1. If $f \in H(\Omega)$ for a compact $\Omega \neq \varnothing$, then there exists $f_{0} \in \boldsymbol{A}(\Delta)$ such that $f_{0}=f$ on $\Delta \cap \Omega_{0}$, for some neighborhood $\Omega_{0}$ of $\Omega$.

This condition excludes in particular the noninteresting case $A(\Delta)=H(\Delta)$. We shall consider here only $\Delta=R$ (the real line) or $\Delta=\boldsymbol{C}$ (the complex plane), and assume that $\boldsymbol{A}_{0}=\{f \in \boldsymbol{A} \mid f$ has compact support $\}$ is dense in $\boldsymbol{A}$.

Fix $f \in \boldsymbol{A}_{0}$. If $g \in H(\operatorname{Spt} f)$, Condition 1 implies the existence of $g_{0} \in \boldsymbol{A}$ such that $g_{0}=g$ on $\operatorname{Spt} f$. The map $M_{f}: H(\operatorname{Spt} f) \rightarrow \boldsymbol{A}$ given by $M_{f} g=f g_{0}$ is well defined.

Condition 2. The map $M_{f}: H(\operatorname{Spt} f) \rightarrow \boldsymbol{A}$ is continuous, for each $f \in \boldsymbol{A}_{0}$. A topological algebra $\boldsymbol{A}$ as in $\$ 1$ which satisfies also Conditions 1 and 2 is called a basic algebra (compare [5]). Example: $C^{n}$ for $0 \leqq n \leqq \infty$.

\section{Restrictions on $T(\cdot)$.}

Condition 3. $T(\cdot)$ has compact support (denoted by $\Sigma$ ). If $g \in H(\Sigma)$ and $g_{0} \in \boldsymbol{A}$ is such that $g_{0}=g$ in a neighborhood of $\Sigma$ (cf. Condition 1),

\footnotetext{
${ }^{1}$ Research partly supported by NSF Grant No. NSF-GP780.
} 
define a representation $T_{H}: H(\Sigma) \rightarrow B(X)$ by $T_{H}(g)=T\left(g_{0}\right) . T_{H}$ is well defined. We call it the restriction of $T(\cdot)$ to $H(\Sigma)$.

Condition 4. $T_{H}: H(\Sigma) \rightarrow B(X)$ is continuous.

Definition. An operational calculus (o.c.) is the object $(\boldsymbol{A}, T(\cdot))$ consisting of a basic algebra $\boldsymbol{A}$ and of a continuous representation $T(\cdot)$ of $\boldsymbol{A}$ in $B(X)$ which satisfies Conditions 3 and 4 as well as the normalizing condition $T(1)=I$. An operator $T$ is of class $\boldsymbol{A}(T \in(\boldsymbol{A}))$ if there exists an o.c. $(\boldsymbol{A}, T(\cdot))$ such that $T(z)=T$.

4. Basic facts. Let $T \in(\boldsymbol{A})$ and let $(\boldsymbol{A}, T(\cdot))$ be an o.c. for $T$. Then

1. $\Sigma=\sigma(T)$ (the spectrum of $T$ ).

2. The restriction of $T(\cdot)$ to $H(\Sigma)$ is the usual analytic operational calculus for $T$.

Property 1 motivates the convention: for real operators (i.e., $\sigma(T) \subset R)$, we take $\Delta=R$.

It is reasonable to require that if $T \in(A)$, also $t T \in(A)$ for $t \in R$. This corresponds to the following requirement on $A$ : if $f \in A$, then $f_{t} \in \boldsymbol{A}$ (where $f_{t}(x)=f(t x)$ ) and the map $f \rightarrow f_{t}$ of $\boldsymbol{A}$ into itself is continuous $(t \in R)$. If $\boldsymbol{A}$ has this property, we say that $\boldsymbol{A}$ is homogeneous.

Theorem 1 ("Classification Theorem"). If $T$ is a real operator of class $\boldsymbol{A}$ for a homogeneous Banach algebra $\boldsymbol{A}$, then $T$ is of class $C^{n}$ for some $n<\infty$.

5. Operators of finite class. We say that $T$ is of finite class if it is of class $C^{n}$ for some $n<\infty$ (cf. Theorem 1).

The proof of the Classification Theorem is based on the following characterization of operators of finite class.

THEOREM 2. T is a real operator of finite class if and only if $\left\|e^{i t T}\right\|$ $=O\left(|t|^{k}\right)(t \in R,|t| \rightarrow \infty)$ for some $0 \leqq k<\infty$.

More precisely, if $T$ is real of class $C^{n}$, then $\left\|e^{i t T}\right\|=O\left(|t|^{n}\right)$; conversely, the latter condition is sufficient for $T$ to be real of class $C^{n+2}$.

6. Relationship with spectral operators. Theorem 2 implies in particular that sums and products of commuting real operators of finite class are of finite class. Spectral operators of finite type (cf. [2]) are operators of finite class. The converse is false by the preceding remark, even in reflexive Banach spaces (cf. [6, pp. 303-304]). However, it is true that $T \in(C)$ if and only if $T$ is spectral of scalar type (if $X$ is weakly complete). In particular, when $X$ is a Hilbert space, $(C)$ is the class of all operators which are similar to normal operators. 
Moreover, $T$ is normal if and only if it is of class $C$ and has a normdecreasing $C$-o.c.

If $T \in\left(C^{n}\right)(1 \leqq n<\infty), x \in X$ and $x^{*} \in X^{*}$, then $x^{*} T(\cdot) x$ is a continuous linear functional on $C^{n}$ with compact support $\Sigma$; as such, it has representations of the form $\sum_{0 \leqq j \leqq n} \mu_{j}^{(j)}$, where $\mu_{j}$ are regular finite Borel measures with supports in an arbitrary neighborhood of $\Sigma$. We say that $T$ is singular if $x^{*} T(\cdot) x$ has such a representation in which $\mu_{j}(j \geqq 1)$ are singular with respect to Lebesgue measure (for all $\left.x, x^{*}\right)$.

THEOREM 3. A real operator on a reflexive Banach space is singular of class $C^{n}(n \geqq 1)$ if and only if it is spectral of type $n$ and its nilpotent part $N$ and resolution of the identity $E(\cdot)$ are such that $x^{*} N E(\cdot) x$ is singular with respect to Lebesgue measure for all $x$ and $x^{*}$.

In other words, singular real operators of class $C^{n}$ have a "Jordan canonical form" $T=S+N$, where $S$ is scalar and real, $N^{n+1}=O$, and $S$ commutes with $N$ (when $X$ is reflexive).

7. Characterizations of $\left(C^{n}\right)$. Theorem 2 gives a simple characterization of $\mathrm{U}_{n \gtrless 0}\left(C^{n}\right)$ in terms of a growth condition on the group $e^{i t T}, t \in R$. In order to characterize in a similar way a given class $\left(C^{n}\right)$, we need some "averages" of $e^{i t T}$. Let $L_{1, n}=\left\{f \in L_{1}(R) \mid t^{i} f(t)\right.$ $\left.\in L_{1}(R) ; 0 \leqq j \leqq n\right\}$; if $f \in L_{1, n}$, its Fourier transform $\hat{f}$ is obviously in $C^{n}$. For $g \in C^{n}$ and $\Delta$ compact, write

$$
\|g\|_{n, \Delta}=\sum_{0 \leqq j \leqq n} \frac{1}{j !} \sup _{\Delta}\left|g^{(j)}\right| .
$$

Definition. Let $n$ be a non-negative integer, $\Delta$ a compact interval, and $T \in B(X)$. The nth variation of $T$ over $\Delta$ is defined by

$$
v_{n}(T ; \Delta)=\sup \left\|\int f(t) e^{i t r} d t\right\|,
$$

where the sup is taken over all $f \in L_{1, n}$ with $\|\hat{f}\|_{n, \Delta}=1 .^{2}$

In general, $v_{n}(T ; \Delta)=\infty$. However, we have

Theorem 4. $T$ is real of class $C^{n}$ if and only if $v_{n}(T ; \Delta)<\infty$ for some compact interval $\Delta$. (In this case, $\sigma(T) \subseteq \Delta$.)

This generalizes Theorem 6 in [4]. As a first corollary, we have the following generalization of results of Bade's [1]:

2 If the integral does not converge in the uniform operator topology for some $f$ in $L_{1, n}$, we set its norm equal to $+\infty$. 
Theorem 5. Let $T_{a} \in B(X)$ be a net converging to $T \in B(X)$ in the strong operator topology. Suppose that, for some $n$ and some compact interval $\Delta, \sup _{a} v_{n}\left(T_{a} ; \Delta\right)<\infty$. Then $T$ (as well as all $\left.T_{a}\right)$ is of class $C^{n}$ with spectrum in $\Delta$, and $T(f)=\lim T_{a}(f)\left(f \in C^{n}\right)$ in the strong operator topology.

Applying Theorem 5, we get

THEOREM 6. Let $T$ and $S$ be two commuting real operators in Hilbert space, $T \in\left(C^{n}\right)$ and $S \in(C)$. Then $T+S \in\left(C^{n}\right)$ and

$$
(T+S)(f)=\int T\left(f_{t}\right) d E(t), \quad f \in C^{n},
$$

where $E(\cdot)$ is the resolution of the identity for $S, f_{t}(x)=f(t+x)$ and the integral exists in the strong operator topology.

An analogous result holds in arbitrary Banach spaces, but it would be too long to state it here. Theorem 6 generalizes known results about spectral operators.

The growth condition in Theorem 4 may be expressed in terms of the resolvent. For example, for $n=0$, we get: A real operator is of class $C$ if and only if the integral $\int\left|x^{*}[T(t-i s ; T)-R(t+i s ; T)] x\right| d t$ is uniformly bounded when $s \rightarrow 0+$, for all unit vectors $x$ and $x^{*}$. In this case, the $C$-o.c. for $T$ is given by

$$
T(f)=\lim _{s \rightarrow 0+} \frac{1}{2 \pi i} \int f(t)[R(t-i s ; T)-R(t+i s ; T)] d t, \quad f \in C .
$$

This explicit representation of the o.c. is well known for hermitian operators (compare [7]). Another explicit representation of the $C^{n}$-o.c., together with a characterization of $\left(C^{n}\right)$, may be obtained as follows. For $u \geqq 0, t \in R, m=1,2, \cdots$, and $T \in B(X)$ arbitrary, let

$$
G_{m}(t, u)=\frac{1}{2 \pi} \int \exp -\left[(v / m)^{2}+u|v|+i v t\right] e^{-i v T} d v
$$

and

$$
T_{m}(f ; u)=\int f(t) G_{m}(t, u) d t, \quad f \in C_{0}^{n},
$$

where $C_{0}^{n}=\left\{f \in C^{n} \mid f\right.$ has compact support $\}$.

Theorem 7. A real operator $T$ is of class $C^{n}$ if and only if, for every $f \in C_{0}^{n}, T_{m}(f ; u) \rightarrow T(f ; u) \in B(X)$ in the weak operator topology $(m \rightarrow \infty)$, 
uniformly with respect to $u(u \geqq 0)$, and, for some constant $M>0$ and some compact interval $\Delta,\|T(f ; u)\| \leqq M\|f\|_{n, \Delta}(u \geqq 0)$.

In this case, the $C^{n}-$ o.c. for $T$ is given by $T(f)=T\left(f_{0} ; 0\right), f \in C^{n}$, where $f_{0} \in C_{0}^{n}$ is such that $f_{0}=f$ on $\Delta$.

\section{REFERENCES}

1. W. G. Bade, Weak and strong limits of spectral operators, Pacific J. Math. 4 (1954), 393-413.

2. N. Dunford, $A$ survey of the theory of spectral operators, Bull. Amer. Math. Soc. 64 (1958), 217-274.

3. C. Foias, Une application des distributions vectorielles d la theorie spectrale, Bull. Sci. Math. (2) 84 (1960), 147-158.

4. S. Kantorovitz, On the characterization of spectral operators, Trans. Amer. Math. Soc. 111 (1964), 152-181.

5. F. Maeda, Generalized spectral operators on locally convex spaces, Pacific J. Math. 13 (1963), 177-192.

6. C. A. McCarthy, Commuting Boolean algebras of projections, Pacific J. Math. 11 (1961), 295-307.

7. H. G. Tillmann, Vector-valued distributions and the spectral theorem for selfadjoint operators in Hilbert space, Bull. Amer. Math. Soc. 69 (1963), 67-71.

Princeton University and

Institute for Advanced Study 